\title{
Managing Complex Systems - When Less is More
}

\author{
Lyman CHAPIN, Bolt Beranek and Newman, U.S.A.
}

In An Introduction to Mathematics, Alfred North Whitehead observes that "Civilization advances by extending the number of important operations which we can perform without thinking about them." That comment was made in 1911, at a time when scientific discovery and its intellectual corollaries were adding so rapidly to the store of human knowledge that Whitehead's generation would be the last to enjoy the expectation of a well-educated man that the compass of his learning include "all things that are known." Highly specialized experts dominate today's science and technology. The complexity of the systems with which we regularly interact, and the sheer quantity of information that clamors for our attention, suggest that civilization has advanced very little during the computer age.

The Internet, to take an obvious example, is surely one of the most successful large-scale distributed enterprises in history; it is used by millions of people in at least 110 countries, and is growing so rapidly that estimates of the "size of the Internet" are obsolete long before they can be published. For even its most sophisticated users, however, the Internet is a dauntingly complex system. Vint Cerf's recent assessment of the state of the Internet is telling: "It's still rocket science." The same could be said of every other large public or private network.

Engineers and other technology specialists tend to view the complexity of networks with the complacency of insiders. The prevailing engineer's viewpoint is dominated by an "engineering meritocracy" ethic that values and rewards "gurus" to whom the secrets of the network have been revealed. The tools and methodologies that are available for managing large networks reflect a corresponding lack of interest in making things simpler - the interesting problems for engineers are elsewhere. As a result, most network management systems exhibit in practice a property that Dave Oran has called the "first law of network management parameters": for every configurable component of a network management system, there are just two settings: the one that works, and all others.

The solution to this problem is <<less >> network management, not more. The last thing a network manager needs is twice as many configurable parameters to set to the wrong values, or a hundred new alerts that report irrelevant or incomprehensible events. The ideal network (from a manager's perspective) would be largely self-configuring and self-managing, requiring very 
little manual intervention. Unfortunately, "manageability" is not high on the list of priorities for most network engineers. A well-designed network management system can compensate for some of the consequences of a poorly-designed (from the standpoint of manageability) network, but often only by requiring the manager to exercise direct control over low-level details. The latest work on object-oriented network management models will be a step forward only if it recognizes reducing complexity as the highest priority. 